\title{
ASYMPTOTICALLY FLAT EXTENSIONS OF CMC BARTNIK DATA
}

\author{
ARMANDO J. CABRERA PACHECO, CARLA CEDERBAUM, STEPHEN MCCORMICK, \\ AND PENGZI MIAO
}

\begin{abstract}
Let $g$ be a metric on the 2 -sphere $\mathbb{S}^{2}$ with positive Gaussian curvature and $H$ be a positive constant. Under suitable conditions on $(g, H)$, we construct smooth, asymptotically flat 3-manifolds $M$ with non-negative scalar curvature, with outer-minimizing boundary isometric to $\left(\mathbb{S}^{2}, g\right)$ and having mean curvature $H$, such that near infinity $M$ is isometric to a spatial Schwarzschild manifold whose mass $m$ can be made arbitrarily close to a constant multiple of the Hawking mass of $\left(\mathbb{S}^{2}, g, H\right)$. Moreover, this constant multiplicative factor depends only on $(g, H)$ and tends to 1 as $H$ tends to 0 . The result provides a new upper bound of the Bartnik mass associated to such boundary data.
\end{abstract}

\section{INTRODUCTION}

Let $g$ be a metric on the 2-sphere $\mathbb{S}^{2}$ for which the first eigenvalue of $-\Delta+K$ is positive, where $K$ is the Gaussian curvature of $g$. Mantoulidis and Schoen [10] constructed asymptotically flat 3-manifolds with non-negative scalar curvature, whose boundaries are outermost minimal surfaces that are isometric to $\left(\mathbb{S}^{2}, g\right)$ and with ADM mass $m_{A D M}$ [1] that can be made arbitrarily close to the optimal value determined by the Riemannian Penrose inequality [4, 8].

In terms of the quasi-local mass $\mathfrak{m}_{B}$ introduced by Bartnik [2, 3], the result of Mantoulidis and Schoen can be reformulated as follows: Given smooth Bartnik data $(\Sigma, g, H)$, i.e., a triple with $\Sigma \simeq \mathbb{S}^{2}, g$ a metric and $H$ a function on $\Sigma$, if $H=0$ and $g$ has positive first eigenvalue $\lambda_{1}(-\Delta+K)>0$, then $\mathfrak{m}_{B}(\Sigma, g, H)$ of such Bartnik data is bounded above by their Hawking mass $\mathfrak{m}_{H}(\Sigma, g, H)$ :

$$
\mathfrak{m}_{B}(\Sigma, g, 0) \leq \mathfrak{m}_{H}(\Sigma, g, 0),
$$

where the Hawking mass $\mathfrak{m}_{H}$ [7] is defined by

$$
\mathfrak{m}_{H}(\Sigma, g, H):=\sqrt{\frac{|\Sigma|_{g}}{16 \pi}}\left(1-\frac{1}{16 \pi} \int_{\Sigma} H^{2} d \sigma\right) .
$$

Bartnik's quasi-local mass $\mathfrak{m}_{B}$ is defined as

$$
\mathfrak{m}_{B}(\Sigma, g, H):=\inf \left\{m_{A D M}(M, \gamma) \mid(M, \gamma) \text { admissible extension of }(\Sigma, g, H)\right\},
$$

where a smooth, asymptotically flat Riemannian 3-manifold $(M, \gamma)$ with boundary $\partial M$ is called an admissible extension of $(\Sigma, g, H)$ if it has non-negative scalar curvature and if $(\Sigma, g)$ is isometric to $\partial M$ with mean curvature $H$. Moreover, it is required 
that $(M, \gamma)$ contains no closed minimal surfaces (except possibly $\partial M$ ) or, a fortiori, that $\partial M$ is outer-minimizing in $(M, \gamma)$, see [4, 5, 8]. Via the proof of the Riemannian Penrose inequality in [8], the outer-minimizing condition allows one to estimate the Bartnik mass of given Bartnik data from below by its Hawking mass.

For the purpose of this paper, it will make no difference which of the above two conditions - no closed minimal surfaces versus outer-minimizing - is chosen as the extensions we construct satisfy both conditions. Here and in the following, we will abbreviate $\mathfrak{m}_{H}(\Sigma):=\mathfrak{m}_{H}(\Sigma, g, H)$ and $\mathfrak{m}_{B}(\Sigma):=\mathfrak{m}_{B}(\Sigma, g, H)$ whenever the choice of $g$ and $H$ is clear from context.

In the important case of $H=0$ treated by Mantoulidis and Schoen, combined with the Riemannian Penrose inequality, (1.1) implies $\mathfrak{m}_{B}(\Sigma, g, 0)=\mathfrak{m}_{H}(\Sigma, g, 0)$. We note that the condition $\lambda_{1}(-\Delta+K)>0$ used in this setting arises naturally in the context of stable minimal surfaces.

In this paper, we give an analogue of Mantoulidis and Schoen's result for a triple of Bartnik data $(\Sigma, g, H)$ that is associated to a constant mean curvature (CMC) surface. As a special case of our main Theorem (Theorem 3.1), we have the following result on constructing asymptotically flat extensions with controlled ADM mass.

Theorem 1.1. Let $\left(\Sigma \simeq \mathbb{S}^{2}, g, H\right)$ be a triple of Bartnik data where $H=H_{o}$ is a positive constant and $g$ has positive Gaussian curvature. There exist constants $\alpha \geq 0$ and $0<\beta \leq 1$, depending on $g$, such that if

$$
\frac{1}{16 \pi} \int_{\Sigma} H_{o}^{2} d \sigma<\frac{\beta}{1+\alpha}
$$

then for each $m>m_{*}$, with

$$
m_{*}=\left[1+\left(\frac{\alpha\left(\frac{1}{16 \pi} \int_{\Sigma} H_{o}^{2} d \sigma\right)}{\beta-(1+\alpha)\left(\frac{1}{16 \pi} \int_{\Sigma} H_{o}^{2} d \sigma\right)}\right)^{\frac{1}{2}}\right] \mathfrak{m}_{H}\left(\Sigma, g, H_{o}\right),
$$

there exists a smooth, asymptotically flat Riemannian 3-manifold $(M, \gamma)$ with boundary $\partial M$ and non-negative scalar curvature such that

(i) $\partial M$ is isometric to $(\Sigma, g)$ and has constant mean curvature $H_{o}$;

(ii) $M$, outside a compact set, is isometric to a spatial Schwarzschild manifold $M_{m}^{S}$ of mass $m$; and

(iii) $M$ is foliated by mean convex 2-spheres which eventually coincide with the rotationally symmetric 2 -spheres in $M_{m}^{S}$.

Remark 1.1. Condition (1.2) implies

$$
\frac{1}{16 \pi} \int_{\Sigma} H_{o}^{2} d \sigma<\frac{\beta}{1+\alpha} \leq 1
$$

hence $\mathfrak{m}_{H}(\Sigma)>0$ is implicitly assumed in Theorem 1.1. Moreover, (iii) implies that $\partial M$ is outer-minimizing in $M$.

Similar to the implication of Mantoulidis and Schoen's result on the Bartnik mass, Theorem 1.1 has the following direct corollary. 
Corollary 1.1. Let $\left(\Sigma \simeq \mathbb{S}^{2}, g, H\right)$ be a triple of Bartnik data where $H=H_{o}$ is a positive constant and $g$ has positive Gaussian curvature. Suppose (1.2) holds, then

$$
\mathfrak{m}_{B}\left(\Sigma, g, H_{o}\right) \leq\left[1+\left(\frac{\alpha\left(\frac{1}{16 \pi} \int_{\Sigma} H_{o}^{2} d \sigma\right)}{\beta-(1+\alpha)\left(\frac{1}{16 \pi} \int_{\Sigma} H_{o}^{2} d \sigma\right)}\right)^{\frac{1}{2}}\right] \mathfrak{m}_{H}\left(\Sigma, g, H_{o}\right) .
$$

Remark 1.2. Inequality (1.3) has the feature that the ratio between the upper bound it provides for $\mathfrak{m}_{B}(\Sigma)$ and the Hawking mass $\mathfrak{m}_{H}(\Sigma)$ tends to 1 as $H_{o} \rightarrow 0$. However, it assumes (1.2). In [9], Lin and Sormani investigated the Bartnik mass of arbitrary CMC Bartnik data $\left(\Sigma, g, H_{o}\right)$, with area $|\Sigma|_{g}=4 \pi$, and proved that

$$
\mathfrak{m}_{B}(\Sigma) \leq \mathfrak{m}_{a \mathbb{S}}(\Sigma)+\mathfrak{m}_{H}(\Sigma)
$$

where $\mathfrak{m}_{a \mathbb{S}}(\Sigma)$, referred to as the asphericity mass, is a non-negative constant that is determined only by the metric $g$ on $\Sigma$. Note that, in contrast to (1.3), the ratio between the right hand side of (1.4) and the Hawking mass approaches a fixed constant $C>1$ as $H \rightarrow 0$.

Remark 1.3. The quantity $m_{*}$ in Theorem 1.1 was first found by Miao and Xie in [12]. Indeed, the Bartnik mass estimate (1.3) would follow from the result in [12] if one allows admissible extensions to be Lipschitz with distributional non-negative scalar curvature across a hypersurface (cf. [11, 13]). Therefore, our main contribution here is to construct smooth extensions.

We will prove Theorem 1.1 applying the method of Mantoulidis and Schoen [10], which involves two steps. In the first step, one needs a collar extension of $\left(\Sigma, g, H_{o}\right)$ with positive scalar curvature on $[0,1] \times \Sigma$ such that the geometric information at $\Sigma_{0}:=\{0\} \times \Sigma$, corresponding to $\left(\Sigma, g, H_{o}\right)$, is suitably propagated to the other end $\Sigma_{1}:=\{1\} \times \Sigma$, near which the extension is rotationally symmetric. (In the minimal surface case, it is primarily the area of $\Sigma_{1}$ that one wants to compare with $\Sigma_{0}$. When $H_{o}>0$, it is the two Hawking masses that one wants to compare.) In the second step, one smoothly glues the collar extension, at $\Sigma_{1}$, to a spatial Schwarzschild manifold (suitably deformed in a small region) with mass greater than, but arbitrarily close to, the Hawking mass of $\Sigma_{1}$.

To implement this process, we make use of the collar extension constructed in [12] in the first step as it provides a good control of the Hawking mass along the collar. For the second step, we prove an elementary result on smoothly gluing a rotationally symmetric manifold with positive scalar curvature and a spatial Schwarzschild manifold with suitably chosen mass (see Proposition 2.1). We give these gluing tools in Section 2, then in Section 3, we prove Theorem 3.1 which implies Theorem 1.1.

Acknowledgements. The work of CC and SM was partially supported by the DAAD and Universities Australia. CC is indebted to the Baden-Württemberg Stiftung for the financial support of this research project by the Eliteprogramme for Postdocs. The work of CC is supported by the Institutional Strategy of the University of Tübingen 
(Deutsche Forschungsgemeinschaft, ZUK 63). The work of PM was partially supported by Simons Foundation Collaboration Grant for Mathematicians \#281105.

\section{Gluing to a spatial Schwarzschild manifold}

In this section, we list some tools for gluing together two rotationally symmetric metrics with non-negative scalar curvature, which are used to prove the main result in Section 3. We start with a lemma that is a slight generalization of [10, Lemma 2.2]. As it may be of independent interest, we state it for arbitrary dimension $n \geq 2$.

Lemma 2.1. Let $f_{i}:\left[a_{i}, b_{i}\right] \rightarrow \mathbb{R}^{+}$, where $i=1,2$, be smooth positive functions, and let $g_{*}$ be the standard metric on $\mathbb{S}^{n}$. Suppose that

(i) the metrics $\gamma_{i}:=d t^{2}+f_{i}(t)^{2} g_{*}$ have positive scalar curvature;

(ii) $f_{1}\left(b_{1}\right)<f_{2}\left(a_{2}\right)$;

(iii) $1>f_{1}^{\prime}\left(b_{1}\right)>0$ and $f_{1}^{\prime}\left(b_{1}\right) \geq f_{2}^{\prime}\left(a_{2}\right)>-1$.

Then, after translating the intervals so that

$$
\left\{\begin{aligned}
\left(a_{2}-b_{1}\right) f_{1}^{\prime}\left(b_{1}\right)=f_{2}\left(a_{2}\right)-f_{1}\left(b_{1}\right), & \text { if } f_{1}^{\prime}\left(b_{1}\right)=f_{2}^{\prime}\left(a_{2}\right), \\
\left(a_{2}-b_{1}\right) f_{1}^{\prime}\left(b_{1}\right)>f_{2}\left(a_{2}\right)-f_{1}\left(b_{1}\right)>\left(a_{2}-b_{1}\right) f_{2}^{\prime}\left(a_{2}\right), & \text { if } f_{1}^{\prime}\left(b_{1}\right)>f_{2}^{\prime}\left(a_{2}\right),
\end{aligned}\right.
$$

one can construct a smooth positive function $f:\left[a_{1}, b_{2}\right] \rightarrow \mathbb{R}^{+}$so that:

(I) $f \equiv f_{1}$ on $\left[a_{1}, \frac{a_{1}+b_{1}}{2}\right], f \equiv f_{2}$ on $\left[\frac{a_{2}+b_{2}}{2}, b_{2}\right]$, and

(II) $\gamma:=d t^{2}+f(t)^{2} g_{*}$ has positive scalar curvature on $\left[a_{1}, b_{2}\right] \times \mathbb{S}^{n}$.

Moreover, if $f_{i}^{\prime}>0$ on $\left[a_{i}, b_{i}\right]$, then $f$ can be constructed such that $f^{\prime}>0$ on $\left[a_{1}, b_{2}\right]$.

Remark 2.1. The assumptions in Lemma 2.1 are weaker than those in [10, Lemma 2.2] employed by Mantoulidis and Schoen. In [10, Lemma 2.2], it was assumed that $f_{i}^{\prime}>0$ and $f_{i}^{\prime \prime}>0$, which implies that $f_{i}^{\prime}<1$ since each $\gamma_{i}$ has positive scalar curvature (cf. (2.6) ); moreover, we relax the assumption $f_{1}^{\prime}\left(b_{1}\right)=f_{2}^{\prime}\left(a_{2}\right)$ in [10, Lemma 2.2] to $f_{1}^{\prime}\left(b_{1}\right) \geq f_{2}^{\prime}\left(a_{2}\right)$, which corresponds to a mean curvature jump condition used in the proof of the positive mass theorem with corners by Miao [11]. This assumption in terms of an inequality helps one simplify the metric gluing procedure used later.

Proof. By (ii) and (iii), the interval $\left[a_{2}, b_{2}\right]$ can always be translated so that (2.1) holds. Assume that such a translation has been performed. On $\left[b_{1}, a_{2}\right]$, (2.1) implies that there exists a function $\zeta \in C^{1}\left(\left[b_{1}, a_{2}\right]\right)$ such that $\zeta\left(b_{1}\right)=f_{1}^{\prime}\left(b_{1}\right), \zeta\left(a_{2}\right)=f_{2}^{\prime}\left(a_{2}\right)$, $\zeta^{\prime} \leq 0$, and $\int_{b_{1}}^{a_{2}} \zeta(t) d t=f_{2}\left(a_{2}\right)-f_{1}\left(b_{1}\right)$. On $\left[b_{1}, a_{2}\right]$, define

$$
\widehat{f}(t):=f_{1}\left(b_{1}\right)+\int_{b_{1}}^{t} \zeta(x) d x
$$


Then $\widehat{f}$ satisfies

$$
\begin{aligned}
\widehat{f}\left(b_{1}\right) & =f_{1}\left(b_{1}\right) \text { and } \widehat{f}\left(a_{2}\right)=f_{2}\left(a_{2}\right), \\
\widehat{f}^{\prime}\left(b_{1}\right) & =f_{1}^{\prime}\left(b_{1}\right) \text { and } \widehat{f}^{\prime}\left(a_{2}\right)=f_{2}^{\prime}\left(a_{2}\right), \\
1>f_{1}^{\prime}\left(b_{1}\right) & \geq \widehat{f}^{\prime}(t) \geq f_{2}^{\prime}\left(a_{2}\right)>-1 \text { on }\left(b_{1}, a_{2}\right), \\
\widehat{f}^{\prime \prime}(t) & =\zeta^{\prime}(t) \leq 0 \text { on }\left[b_{1}, a_{2}\right] .
\end{aligned}
$$

On $\left[a_{1}, b_{2}\right]$, define

$$
\widetilde{f}(t):= \begin{cases}f_{1}(t) & \text { on }\left[a_{1}, b_{1}\right] \\ \widehat{f}(t) & \text { on }\left[b_{1}, a_{2}\right] \\ f_{2}(t) & \text { on }\left[a_{2}, b_{2}\right]\end{cases}
$$

Then $\tilde{f} \in C^{1,1}\left(\left[a_{1}, b_{2}\right]\right), C^{2}$ away from $b_{1}, a_{2}$, and $\tilde{f}>0$. Slightly abusing notation, we will write $\tilde{f}^{\prime \prime}$ on all of $\left[a_{1}, b_{2}\right]$ extending it to $b_{1}, a_{2}$ by setting $\widetilde{f}^{\prime \prime}\left(b_{1}\right):=f_{1}^{\prime \prime}\left(b_{1}\right)$ and $\widetilde{f}^{\prime \prime}\left(a_{2}\right):=f_{2}^{\prime \prime}\left(a_{2}\right)$.

We next consider an appropriate mollification of $\tilde{f}$ (cf. [6]). Let $\delta>0$ be such that

$$
\frac{a_{1}+b_{1}}{2}<b_{1}-\delta \text { and } a_{2}+\delta<\frac{a_{2}+b_{2}}{2}
$$

Let $\eta_{\delta}:\left[a_{1}, b_{2}\right] \rightarrow \mathbb{R}_{0}^{+}$be a smooth cutoff function which equals 1 on $\left[b_{1}-\delta, a_{2}+\delta\right]$, vanishes on $\left[a_{1}, \frac{a_{1}+b_{1}}{2}\right] \cup\left[\frac{a_{2}+b_{2}}{2}, b_{2}\right]$, and satisfies $0<\eta_{\delta}(s)<1$ in the remaining part of the interval. Let $\phi: \mathbb{R} \rightarrow \mathbb{R}_{0}^{+}$be a standard smooth mollifier with compact support in $[-1,1]$ and $\int_{-\infty}^{\infty} \phi(s) d s=1$.

For each positive $\varepsilon<\frac{\delta}{4}$, define $f_{\varepsilon}$ by

$$
f_{\varepsilon}(t):=\int_{-\infty}^{\infty} \widetilde{f}\left(t-\varepsilon \eta_{\delta}(t) s\right) \phi(s) d s, \quad t \in\left[a_{1}, b_{2}\right]
$$

and observe that $f_{\varepsilon}$ is smooth on $\left[a_{1}, b_{2}\right]$. Then $f_{\varepsilon} \equiv \tilde{f}$ on $\left[a_{1}, \frac{a_{1}+b_{1}}{2}\right] \cup\left[\frac{a_{2}+b_{2}}{2}, b_{2}\right]$ and

$$
f_{\varepsilon}^{\prime}(t)=\int_{-\infty}^{\infty} \widetilde{f^{\prime}}\left(t-\varepsilon \eta_{\delta}(t) s\right)\left(1-\varepsilon \eta_{\delta}^{\prime}(t) s\right) \phi(s) d s \forall t \in\left[a_{1}, b_{2}\right] .
$$

Moreover, since $\tilde{f}^{\prime}$ is $C^{0}$ everywhere and $C^{1}$ except at $b_{1}$ and $a_{2}$, it can be checked that by standard mollification arguments

$$
\begin{aligned}
f_{\varepsilon}^{\prime \prime}(t) & =\frac{d}{d t}\left(\int_{-\infty}^{\infty} \widetilde{f}^{\prime}(t-\varepsilon s) \phi(s) d s,\right) \\
& =\int_{-\infty}^{\infty} \widetilde{f}^{\prime \prime}(s) \phi_{\varepsilon}(t-s) d s \quad \forall t \in\left(b_{1}-\delta, a_{2}+\delta\right),
\end{aligned}
$$


where $\phi_{\varepsilon}(t):=\frac{1}{\varepsilon} \phi\left(\frac{t}{\varepsilon}\right)$, and

$$
\begin{aligned}
f_{\varepsilon}^{\prime \prime}(t)= & \int_{-\infty}^{\infty} \tilde{f}^{\prime \prime}\left(t-\varepsilon \eta_{\delta}(t) s\right)\left(1-\varepsilon \eta_{\delta}^{\prime}(t) s\right)^{2} \phi(s) d s \\
& -\varepsilon \int_{-\infty}^{\infty} \widetilde{f}^{\prime}\left(t-\varepsilon \eta_{\delta}(t) s\right) \eta_{\delta}^{\prime \prime}(t) s \phi(s) d s, \forall t \notin\left[b_{1}-\frac{1}{4} \delta, a_{2}+\frac{1}{4} \delta\right] .
\end{aligned}
$$

We claim that, for sufficiently small $\varepsilon>0$, the metric $d t^{2}+f_{\varepsilon}(t)^{2} g_{*}$ has positive scalar curvature. To shows this, recall that given any smooth function $f>0$, the metric $d t^{2}+f(t)^{2} g_{*}$ has positive scalar curvature if and only if

$$
f^{\prime \prime}(t)<\frac{n-1}{2 f(t)}\left(1-f^{\prime}(t)^{2}\right) \quad \forall t .
$$

Suggested by (2.6), given any positive $f \in C^{1}\left(\left[a_{1}, b_{2}\right]\right)$, we define

$$
\Omega[f](t):=\frac{n-1}{2 f(t)}\left(1-f^{\prime}(t)^{2}\right), \quad t \in\left[a_{1}, b_{2}\right] .
$$

Observe that $\Omega[f] \in C^{0}\left(\left[a_{1}, b_{2}\right]\right)$. Then, on $\left[a_{1}, b_{2}\right] \backslash\left\{b_{1}, a_{2}\right\}$, we have

$$
\Omega[\tilde{f}]>\widetilde{f}^{\prime \prime}
$$

because $\Omega[\tilde{f}]>0$ on $\left[b_{1}, a_{2}\right], \widetilde{f}^{\prime \prime} \leq 0$ on $\left(b_{1}, a_{2}\right)$, and $g_{i}$ has positive scalar curvature on $\left[a_{i}, b_{i}\right] \times \mathbb{S}^{n}$ for $i=1,2$. Moreover, for the same reason, we indeed have

$$
3 d:=\inf _{t \in\left[a_{1}, b_{2}\right] \backslash\left\{b_{1}, a_{2}\right\}}\left(\Omega[\tilde{f}]-\widetilde{f}^{\prime \prime}\right)>0 .
$$

Thus,

$$
\tilde{f}^{\prime \prime}(t) \leq \Omega[\tilde{f}](t)-3 d
$$

wherever $\tilde{f}^{\prime \prime}$ is defined. Now we consider $\Omega\left[f_{\varepsilon}\right]$. By (2.2) and (2.3), $f_{\varepsilon} \rightarrow \tilde{f}$ in $C^{1}\left(\left[a_{1}, b_{2}\right]\right)$ as $\varepsilon \rightarrow 0^{+}$. Hence, $\Omega\left[f_{\varepsilon}\right] \rightarrow \Omega[\widetilde{f}]$ in $C^{0}\left(\left[a_{1}, b_{2}\right]\right)$, which shows, for small $\varepsilon$,

$$
\sup _{t \in\left[a_{1}, b_{2}\right]}\left|\Omega[\tilde{f}](t)-\Omega\left[f_{\varepsilon}\right](t)\right|<d .
$$

Moreover, by (2.4) and (2.5),

$$
f_{\varepsilon}^{\prime \prime}(t)<\sup _{|s-t|<\varepsilon} \tilde{f}^{\prime \prime}(s)+d \quad \forall t \in\left[a_{1}, b_{2}\right]
$$

provided $\varepsilon$ is sufficiently small. Therefore, it follows that, for all $t \in\left[a_{1}, b_{2}\right]$,

$$
\begin{aligned}
f_{\varepsilon}^{\prime \prime}(t) & \leq \sup _{|s-t|<\varepsilon}(\Omega[\tilde{f}](s)-3 d)+d \\
& <\Omega[\tilde{f}](t)-d \\
& <\Omega\left[f_{\varepsilon}\right](t)
\end{aligned}
$$


where, in the second to last inequality, we also used the fact that $\Omega[\tilde{f}]$ is uniformly continuous on $\left[a_{1}, b_{2}\right]$, and hence $\Omega[\tilde{f}](s)<\Omega[\tilde{f}](t)+d$ for any $s$ with $|s-t|<\varepsilon$ provided $\varepsilon$ is small.

Thus, we have shown that (2.6) holds on $\left[a_{1}, b_{2}\right]$ with $f$ replaced by $f_{\varepsilon}$ for small $\varepsilon$. Hence, the metric $d t^{2}+f_{\varepsilon}(t)^{2} g_{*}$ has positive scalar curvature and thus $f:=f_{\varepsilon}$ satisfies the conclusions of the theorem for small enough $\varepsilon$. Finally, if it is assumed that $f_{i}^{\prime}>0$, then $\tilde{f}^{\prime}>0$ which directly implies $f^{\prime}=f_{\varepsilon}^{\prime}>0$ as $f_{\varepsilon} \rightarrow \widetilde{f}$ in $C^{1}\left(\left[a_{1}, b_{2}\right]\right)$.

Remark 2.2. It follows from the above proof that, if $f_{1}^{\prime}\left(b_{1}\right)>f_{2}^{\prime}\left(a_{2}\right)$, the assumptions $f_{1}^{\prime}\left(b_{1}\right)<1$ and $f_{2}^{\prime}\left(a_{2}\right)>-1$ can be weakened to $f_{1}^{\prime}\left(b_{1}\right) \leq 1$ and $f_{2}^{\prime}\left(a_{2}\right) \geq-1$, respectively. This is because, in this case, one can require $\zeta^{\prime}(t)<0$ on $\left[b_{1}, a_{2}\right]$. Thus, $\widehat{f}^{\prime \prime} \leq \max _{\left[b_{1}, a_{2}\right]} \zeta^{\prime}<0$ on $\left(b_{1}, a_{2}\right)$ while $\Omega[\widetilde{f}] \geq 0$ on $\left[b_{1}, a_{2}\right]$. Therefore, (2.7) still holds.

Proposition 2.1. Consider a metric $\gamma=d s^{2}+f(s)^{2} g_{*}$ on $[a, b] \times \mathbb{S}^{2}$, where $g_{*}$ is the standard metric on $\mathbb{S}^{2}$ and $f>0$ is a smooth function on $[a, b]$. Suppose

(1) $\gamma$ has positive scalar curvature;

(2) $\Sigma_{b}:=\{b\} \times \mathbb{S}^{2}$ has positive mean curvature; and

(3) $\mathfrak{m}_{H}\left(\Sigma_{b}\right) \geq 0$.

Then, for any $m_{e}>\mathfrak{m}_{H}\left(\Sigma_{b}\right)$, there exists a smooth, rotationally symmetric, asymptotically flat Riemannian 3-manifold $M$ with boundary $\partial M$ and with non-negative scalar curvature such that

(i) $M$, outside a compact set, is isometric to a spatial Schwarzschild manifold of mass $m_{e}$;

(ii) $\partial M$ has a neighborhood $U$ that is isometric to $\left(\left[a, \frac{a+b}{2}\right) \times \mathbb{S}^{2}, \gamma\right)$; and

(iii) if $f^{\prime}>0$ on $[a, b]$, then $M$ can be constructed such that every rotationally symmetric sphere in $M$ has positive constant mean curvature.

To prove this proposition, we recall that a spatial Schwarzschild manifold $\left(M_{m}^{S}, \gamma_{m}\right)$ with mass $m>0$ takes the form of

$$
\left(M_{m}^{S}, \gamma_{m}\right)=\left((2 m, \infty) \times \mathbb{S}^{2}, \frac{1}{1-\frac{2 m}{r}} d r^{2}+r^{2} g_{*}\right)
$$

Setting $s:=\int_{2 m}^{r}\left(1-\frac{2 m}{t}\right)^{-\frac{1}{2}} d t$, we can slightly extend $\left(M_{m}^{S}, \gamma_{m}\right)$ to

$$
\left(M_{m}^{S}, \gamma_{m}\right)=\left([0, \infty) \times \mathbb{S}^{2}, d s^{2}+\bar{u}_{m}^{2}(s) g_{*}\right)
$$

where $\bar{u}_{m}:[0, \infty) \rightarrow[2 m, \infty)$ satisfies $\bar{u}_{m}(0)=2 m$,

$$
\bar{u}_{m}^{\prime}(s)=\sqrt{1-\frac{2 m}{\bar{u}_{m}(s)}} \text { and } \bar{u}_{m}^{\prime \prime}(s)=\frac{m}{\bar{u}_{m}(s)^{2}} .
$$

Proof of Proposition 2.1. The Hawking mass of $\Sigma_{b}$ is given by

$$
\mathfrak{m}_{H}\left(\Sigma_{b}\right)=\frac{f(b)}{2}\left(1-f^{\prime}(b)^{2}\right) .
$$


For simplicity, we set $m_{*}:=\mathfrak{m}_{H}\left(\Sigma_{b}\right), r_{*}:=f(b)$ and $w_{*}:=f^{\prime}(b)$, so (2.11) becomes $m_{*}=\frac{r_{*}}{2}\left(1-w_{*}^{2}\right)$. By assumption, $w_{*}>0$ and $m_{*} \geq 0$. The latter implies $w_{*} \leq 1$.

Given any $m_{e}>m_{*}$, we claim that there there exists $s_{m_{e}}>0$ such that

$$
\begin{aligned}
& \bar{u}_{m_{e}}^{\prime}\left(s_{m_{e}}\right) \leq w_{*}, \\
& \bar{u}_{m_{e}}\left(s_{m_{e}}\right)>r_{*} .
\end{aligned}
$$

In case $m_{*}>0$, i.e., $0<w_{*}<1$, this is easily seen by choosing $s_{m_{e}}>0$ so that $\bar{u}_{m_{e}}^{\prime}\left(s_{m_{e}}\right)=w_{*}$. Then (2.13) follows from (2.10) and the fact that $m_{e}>m_{*}$. If $m_{*}=0$, i.e., $w_{*}=1$, then (2.12) holds (with a strict inequality) for any $s_{m_{e}}>0$. In this case, (2.13) holds for any sufficiently large $s_{m_{e}}$.

To proceed, we fix $s_{m_{e}}$. Let $\delta \in\left(0, s_{m_{e}}\right)$ be a small constant to be chosen later. We now slightly bend a small piece of the Schwarzschild manifold $\left(M_{m_{e}}^{S}, \gamma_{m_{e}}\right)$, near $s=s_{m_{e}}$, so that it has positive scalar curvature and can be smoothly glued here to $\left([a, b] \times \mathbb{S}^{2}, \gamma\right)$ using Lemma 2.1.

Following Lemma 2.3 in [10], we consider a smooth function $\sigma:\left[s_{m_{e}}-\delta, \infty\right) \rightarrow \mathbb{R}$ defined by $\sigma(s):=s$ for $s \geq s_{m_{e}}$, and indirectly via

$$
\sigma^{\prime}(s):=1+e^{-1 /\left(s-s_{m_{e}}\right)^{2}} \text { for } s_{m_{e}}-\delta \leq s \leq s_{m_{e}} .
$$

If $\delta$ is small enough then $\sigma(s)>0$ and the metric

$$
\gamma_{e}:=d s^{2}+\bar{u}_{m_{e}}(\sigma(s))^{2} g_{*}
$$

has positive scalar curvature on $\left[s_{m_{e}}-\delta, s_{m_{e}}\right) \times \mathbb{S}^{2}$. Note that $\gamma_{e}$ is identically the Schwarzschild metric with mass $m_{e}$ on $\left[s_{m_{e}}, \infty\right) \times \mathbb{S}^{2}$ and $\frac{d}{d s} \bar{u}_{m_{e}}(\sigma(s))>0, \forall s \geq$ $s_{m_{e}}-\delta$.

We now want to compare $\left.\bar{u}_{m_{e}}(\sigma(s))\right|_{s=s_{m_{e}}-\delta}$ and $\left.\frac{d}{d s} \bar{u}_{m_{e}}(\sigma(s))\right|_{s=s_{m_{e}}-\delta}$, with $r_{*}$ and $w_{*}$, respectively. By (2.13), we have

$$
\left.\bar{u}_{m_{e}}(\sigma(s))\right|_{s=s_{m_{e}}-\delta}>r_{*}
$$

if $\delta$ is sufficiently small. By (2.10), one finds

$$
\frac{d^{2}}{d s^{2}} \bar{u}_{m_{e}}(\sigma(s))=m_{e} \bar{u}_{m_{e}}(\sigma(s))^{-2} \sigma^{\prime}(s)^{2}+\bar{u}_{m_{e}}^{\prime}(\sigma(s)) \sigma^{\prime \prime}(s) .
$$

Hence $\left.\frac{d^{2}}{d s^{2}} \bar{u}_{m_{e}}(\sigma(s))\right|_{s=s_{m_{e}}}=m_{e} \bar{u}_{m_{e}}\left(s_{m_{e}}\right)^{-2}>0$ since $m_{e}>0$. Thus, shrinking $\delta$ if necessary, we have $\frac{d^{2}}{d s^{2}} \bar{u}_{m_{e}}(\sigma(s))>0$ on $\left[s_{m_{e}}-\delta, s_{m_{e}}\right]$. Combined with (2.12), this implies

$$
\left.\frac{d}{d s} \bar{u}_{m_{e}}(\sigma(s))\right|_{s=s_{m_{e}-\delta}}<\bar{u}_{m_{e}}^{\prime}\left(s_{m_{e}}\right) \leq w_{*} .
$$

Now, by (2.14), (2.15) and as $\gamma_{e}$ has positive scalar curvature on $\left[s_{m_{e}}-\delta, s_{m_{e}}\right) \times \mathbb{S}^{2}$, we can apply Lemma 2.1 (and Remark 2.2) to smoothly glue $\gamma$ and $\gamma_{e}$ together, with $f$ playing the role of $f_{1}$ on $\left[a_{1}, b_{1}\right]:=[a, b]$, and $\bar{u}_{m_{e}} \circ \sigma$ playing the role of $f_{2}$ on $\left[a_{2}, b_{2}\right]:=\left[s_{m_{e}}-\delta, s_{m_{e}}-\frac{\delta}{2}\right]$. The resulting manifold satisfies all properties we require. 
Remark 2.3. We would like to comment on the size of the "neck" that is needed in the above proof to connect $\left([a, b] \times \mathbb{S}^{2}, \gamma\right)$ to $\left(M_{m_{e}}^{S}, \gamma_{m_{e}}\right)$. Precisely, this means that we want to estimate $l:=a_{2}-b_{1}$ after having set $\left[a_{1}, b_{1}\right]=[a, b],\left[a_{2}, b_{2}\right]=\left[s_{m_{e}}-\delta, s_{m_{e}}-\frac{\delta}{2}\right]$, and having translated the intervals so that (2.1) holds. By (2.1) and (2.15), $l$ satisfies

$$
\frac{\bar{u}_{m_{e}}\left(\sigma\left(s_{m_{e}}-\delta\right)\right)-r_{*}}{\left.\frac{d}{d s} \bar{u}_{m_{e}}(\sigma(s))\right|_{s=s_{m_{e}}-\delta}}>l>\frac{\bar{u}_{m_{e}}\left(\sigma\left(s_{m_{e}}-\delta\right)\right)-r_{*}}{w_{*}} .
$$

Suppose $\mathfrak{m}_{H}\left(\Sigma_{b}\right)>0$, by construction we have $\bar{u}_{m_{e}}^{\prime}\left(s_{m_{e}}\right)=w_{*}$ and consequently

$$
\begin{aligned}
& \lim _{\delta \rightarrow 0} \frac{\bar{u}_{m_{e}}\left(\sigma\left(s_{m_{e}}-\delta\right)\right)-r_{*}}{\left.\frac{d}{d s} \bar{u}_{m_{e}}(\sigma(s))\right|_{s=s_{m_{e}}-\delta}} \\
= & \frac{\bar{u}_{m_{e}}\left(s_{m_{e}}\right)-r_{*}}{\bar{u}_{m_{e}}^{\prime}\left(s_{m_{e}}\right)}=\frac{\bar{u}_{m_{e}}\left(s_{m_{e}}\right)-r_{*}}{w_{*}} \\
= & \lim _{\delta \rightarrow 0} \frac{\bar{u}_{m_{e}}\left(\sigma\left(s_{m_{e}}-\delta\right)\right)-r_{*}}{w_{*}} .
\end{aligned}
$$

Thus, by choosing $\delta$ small, we see that $l$ is arbitrarily close to $L:=\frac{\bar{u}_{m_{e}}\left(s_{m_{e}}\right)-r_{*}}{w_{*}}$. On the other hand, we have

$$
L=\frac{\left(\frac{m_{e}}{m_{*}}-1\right) r_{*}}{w_{*}},
$$

which follows from the fact $m_{*}=\frac{r_{*}}{2}\left(1-w_{*}^{2}\right), m_{e}=\frac{1}{2} \bar{u}_{m_{e}}\left(s_{m_{e}}\right)\left(1-\bar{u}_{m_{e}}^{\prime}\left(s_{m_{e}}\right)^{2}\right)$ and $\bar{u}_{m_{e}}^{\prime}\left(s_{m_{e}}\right)=w_{*}$. Therefore, we conclude that $l \rightarrow 0$ as $m_{e} \rightarrow \mathfrak{m}_{H}\left(\Sigma_{b}\right)$.

When $\mathfrak{m}_{H}\left(\Sigma_{b}\right)=0$, i.e. $w_{*}=1, l$ can always be chosen to lie in

$$
\left(\frac{\bar{u}_{m_{e}}\left(s_{m_{e}}\right)-r_{*}}{\bar{u}_{m_{e}}^{\prime}\left(s_{m_{e}}\right)}, \bar{u}_{m_{e}}\left(s_{m_{e}}\right)-r_{*}\right) \text {. }
$$

In this case, by choosing $s_{m_{e}}$ such that $\bar{u}_{m_{e}}\left(s_{m_{e}}\right)$ is arbitrarily close to $r_{*}$, we see that $l$ can be taken to approach 0 for any given $m_{e}>\mathfrak{m}_{H}\left(\Sigma_{b}\right)$.

\section{Asymptotically flat extensions}

Throughout this section, let $\left(\Sigma \simeq \mathbb{S}^{2}, g, H\right)$ be a triple of Bartnik data with $K(g)>$ 0 and constant mean curvature $H=H_{o}>0$. Let $r_{o}$ be the area radius of $g$, i.e., $|\Sigma|_{g}=4 \pi r_{o}^{2}$. We always let $\{g(t)\}_{0 \leq t \leq 1}$ be a smooth path of metrics on $\Sigma$ such that

(i) if $g$ is a round metric, $\{g(t)\}_{0 \leq t \leq 1}$ is the constant path with $g(t)=g, \forall t \in[0,1]$; and

(ii) if $g$ is not a round metric, $\{g(t)\}_{0 \leq t \leq 1}$ is a path of metrics with positive Gaussian curvature satisfying $g(0)=g, g(1)$ is a round metric, and

$$
\operatorname{tr}_{g(t)} g^{\prime}(t)=0, \forall t \in[0,1] \text {. }
$$

(Existence of such a $\{g(t)\}_{0 \leq t \leq 1}$ is given by Mantoulidis and Schoen's proof of [10, Lemma 1.2].) 
As in [12, Section 2], we let $\alpha$ and $\beta$ be two constants determined by such a path via

$$
\begin{aligned}
\alpha & :=\frac{1}{4} \max _{t \in[0,1]} \max _{\Sigma}\left|g^{\prime}(t)\right|_{g(t)}^{2}, \\
\beta & :=r_{o}^{2} \min _{t \in[0,1]} \min _{\Sigma} K(g(t)) .
\end{aligned}
$$

Clearly,

(a) $\beta=1$ and $\alpha=0$, if $g$ is a round metric; and

(b) $0<\beta<1$ and $\alpha>0$, if $g$ is not a round metric.

In assertion (b), one uses the Gauß-Bonnet Theorem and the fact $|\Sigma|_{g(t)}=4 \pi r_{o}^{2}, \forall t$.

The following theorem illustrates how the gluing tools in the previous section and the collar extension in [12] are combined to produce asymptotically flat extensions with suitable CMC Bartnik data.

Theorem 3.1. Let $\left(\Sigma \simeq \mathbb{S}^{2}, g, H\right)$ be a triple of Bartnik data where $g$ has positive Gaussian curvature and $H=H_{o}$ is a positive constant. Let $r_{o}$ be the area radius of g, i.e., $|\Sigma|_{g}=4 \pi r_{o}^{2}$. Let $\{g(t)\}_{0 \leq t \leq 1}$ be a path of metrics given in (i) or (ii) above. Let $\alpha \geq 0$ and $0<\beta \leq 1$ be the constants defined in (3.1) for this path $\{g(t)\}_{0 \leq t \leq 1}$. Suppose the condition

$$
\frac{1}{4} H_{o}^{2} r_{o}^{2}<\frac{\beta}{1+\alpha}
$$

holds. Given any $m \in\left(-\infty, \frac{1}{2} r_{o}\right)$ satisfying

$$
\frac{1}{4} H_{o}^{2} r_{o}^{2}<\frac{\beta}{1+\alpha}\left(1-\frac{2 m}{r_{o}}\right)
$$

let $k>0$ be the constant given by

$$
k:=\frac{H_{o} r_{o}}{2}\left(1-\frac{2 m}{r_{o}}\right)^{-\frac{1}{2}} .
$$

Define

$$
m_{*}:= \begin{cases}\frac{1}{2} r_{o}\left[\frac{\frac{1}{4} H_{o}^{2} r_{o}^{2} \alpha}{\left(\beta-\frac{1}{4} H_{o}^{2} r_{o}^{2} \alpha\right)-k^{2}}\right]^{\frac{1}{2}}\left(1-k^{2}\right)+\mathfrak{m}_{H}(\Sigma), & \text { if } m<0, \\ \frac{1}{2} r_{o}\left[\frac{\alpha k^{2}}{\beta-(1+\alpha) k^{2}}\right]^{\frac{1}{2}}\left(1-k^{2}\right)+\mathfrak{m}_{H}(\Sigma), & \text { if } m \geq 0,\end{cases}
$$

where $\mathfrak{m}_{H}(\Sigma):=\mathfrak{m}_{H}\left(\Sigma, g, H_{o}\right)$. Then, for any $m_{e}>m_{*}$, there exists a smooth, asymptotically flat Riemannian 3-manifold $(M, \gamma)$ with boundary $\partial M$ and non-negative scalar curvature such that

(i) $\partial M$ is isometric to $(\Sigma, g)$ and has constant mean curvature $H_{o}$;

(ii) $M$, outside a compact set, is isometric to a spatial Schwarzschild manifold $M_{m_{e}}^{S}$ of mass $m_{e}$; and 
(iii) $M$ is foliated by mean convex 2 -spheres which eventually coincide with the rotationally symmetric 2 -spheres in $M_{m_{e}}^{S}$.

Proof. We note that (3.2) implies

$$
\frac{1}{16 \pi} \int_{\Sigma} H_{o}^{2} d \sigma<\frac{\beta}{1+\alpha} \leq 1
$$

that is $\mathfrak{m}_{H}(\Sigma)>0$ by hypothesis.

We first consider the case that $g$ is not a round metric, i.e. $\alpha>0$. In this case, we will first prove the theorem under the additional assumption that

$$
g(t)=g(1), \forall t \in[1-\theta, 1]
$$

for some $\theta \in\left(0, \frac{1}{3}\right)$. Such a condition is imposed so that later we can directly apply Proposition 2.1.

Now we describe the collar extension produced in [12, Proposition 2.1]. Given the constant $m \in\left(-\infty, \frac{1}{2} r_{o}\right)$, consider part of the spatial Schwarzschild metric

$$
\gamma_{m}=\frac{1}{1-\frac{2 m}{r}} d r^{2}+r^{2} g_{*}
$$

defined on $\left[r_{o}, \infty\right) \times \mathbb{S}^{2}$. By a change of variable $s:=\int_{r_{o}}^{r}\left(1-\frac{2 m}{t}\right)^{-\frac{1}{2}} d t$, one can write

$$
\gamma_{m}=d s^{2}+u_{m}^{2}(s) g_{*},
$$

where $s \in[0, \infty)$ and $u_{m}$ is the inverse function of $s=s(r)$. (Note that, in the case of $m \geq 0, u_{m}$ is related to $\bar{u}_{m}$ in (2.9) by $u_{m}(t):=\bar{u}_{m}\left(s_{0}+t\right)$, where $s_{0}>0$ is given by $\bar{u}_{m}\left(s_{0}\right)=r_{o}$.) Next, by $(3.2)-(3.4)$, we have

$$
\begin{cases}\beta-\left[1+\left(1-\frac{2 m}{r_{o}}\right) \alpha\right] k^{2}>0, & \text { if } m<0 \\ \beta-(1+\alpha) k^{2}>0, & \text { if } m \geq 0 .\end{cases}
$$

Therefore, we can define a constant $A_{o}>0$ by

$$
A_{o}:= \begin{cases}r_{o}\left[\frac{\alpha}{\beta-\left[1+\left(1-\frac{2 m}{r_{o}}\right) \alpha\right] k^{2}}\right]^{\frac{1}{2}}, & \text { if } m<0 \\ r_{o}\left[\frac{\alpha}{\beta-(1+\alpha) k^{2}}\right]^{\frac{1}{2}}, & \text { if } m \geq 0 .\end{cases}
$$

Applying Proposition 2.1 of [12] (and the subsequent Remark 2.1), we know the metric

$$
\gamma:=A_{o}^{2} d t^{2}+\frac{u_{m}\left(A_{o} k t\right)^{2}}{r_{o}^{2}} g(t)
$$

defined on $N:=[0,1] \times \Sigma$ satisfies:

(i) $R(\gamma)>0$, where $R(\gamma)$ is the scalar curvature of $\gamma$;

(ii) the induced metric on $\Sigma_{0}:=\{0\} \times \Sigma$ by $\gamma$ is $g$; 
(iii) the mean curvature $H(0)$ of $\Sigma_{0}$ is $H(0)=H_{o}$;

(iv) $\Sigma_{t}:=\{t\} \times \mathbb{S}^{2}$ has positive constant mean curvature $\forall t \in[0,1]$; and

(v) the Hawking mass of $\Sigma_{1}=\{1\} \times \Sigma$ is given by

$$
\mathfrak{m}_{H}\left(\Sigma_{1}\right)=\frac{1}{2}\left[u_{m}\left(A_{o} k\right)-r_{o}\right]\left(1-k^{2}\right)+\mathfrak{m}_{H}(\Sigma) .
$$

Moreover, as in [12, Section 3], one can estimate $\mathfrak{m}_{H}\left(\Sigma_{1}\right)$ by

$$
\mathfrak{m}_{H}\left(\Sigma_{1}\right) \leq \begin{cases}\frac{1}{4} H_{o} r_{o} A_{o}\left(1-k^{2}\right)+\mathfrak{m}_{H}(\Sigma) & \text { if } m<0 \\ \frac{1}{2} A_{o} k\left(1-k^{2}\right)+\mathfrak{m}_{H}(\Sigma) & \text { if } m \geq 0 .\end{cases}
$$

Here

$$
\frac{1}{4} H_{o} r_{o} A_{o}=\frac{1}{2} r_{o}\left[\frac{\frac{1}{4} H_{o}^{2} r_{o}^{2} \alpha}{\left(\beta-\frac{1}{4} H_{o}^{2} r_{o}^{2} \alpha\right)-k^{2}}\right]^{\frac{1}{2}} \text { if } m<0,
$$

and

$$
\frac{1}{2} A_{o} k=\frac{1}{2} r_{o}\left[\frac{\alpha k^{2}}{\beta-(1+\alpha) k^{2}}\right]^{\frac{1}{2}} \text { if } m \geq 0 .
$$

In other words, (3.8) is simply to assert

$$
\mathfrak{m}_{H}\left(\Sigma_{1}\right) \leq m_{*} .
$$

By (3.3) and (3.4), we have $k^{2}<\frac{\beta}{1+\alpha}<1$. Therefore,

$$
0<\mathfrak{m}_{H}(\Sigma)<\mathfrak{m}_{H}\left(\Sigma_{1}\right) \leq m_{*} .
$$

Upon a change of variable $s:=A_{o} t,(N, \gamma)$ becomes

$$
\left(\left[0, A_{o}\right] \times \Sigma, d s^{2}+\frac{f(s)^{2}}{r_{o}^{2}} g\left(A_{o}^{-1} s\right)\right),
$$

where $f(s)=u_{m}(k s)$ and $f^{\prime}(s)>0$. On $\left[(1-\theta) A_{o}, A_{o}\right] \times \Sigma$, by (3.6),

$$
\gamma=d s^{2}+f(s)^{2} g_{*}
$$

for some fixed round metric $g_{*}$ of area $4 \pi$. The theorem now follows readily from Proposition 2.1.

Next, we show that the theorem still holds when condition (3.6) is removed. The idea is to simply approximate $\{g(t)\}_{0 \leq t \leq 1}$ by a path satisfying (3.6). Given any small $\theta \in\left(0, \frac{1}{3}\right)$, let $\xi_{\theta}(t) \geq 0$ be a smooth function on $[0,1]$ such that

$$
\left\{\begin{array}{l}
\xi_{\theta}(t)=\frac{t}{1-2 \theta}, \forall t \in[0,1-3 \theta], \\
\xi_{\theta}(t)=1, \forall t \in[1-\theta, 1], \\
0 \leq \xi_{\theta}^{\prime}(t) \leq \frac{1}{1-2 \theta} \forall t \in[0,1] .
\end{array}\right.
$$


(For instance, such an $\xi_{\theta}(t)$ can be obtained by a usual mollification of the piecewise smooth function that equals $\frac{t}{1-2 \theta}$ on $[0,1-2 \theta]$ and equals 1 on $[1-2 \theta, 1]$.) Consider the reparametrized path

$$
g_{\theta}(t):=g\left(\xi_{\theta}(t)\right)
$$

which satisfies $g_{\theta}(t)=g(1)$ for $t \in[1-\theta, 1]$. Let $\alpha_{\theta}$ and $\beta_{\theta}$ be the corresponding constants defined in (3.1) with $g(t)$ replaced by $g_{\theta}(t)$. Clearly,

$$
\beta_{\theta}=\beta \text {. }
$$

We claim

$$
\lim _{\theta \rightarrow 0} \alpha_{\theta}=\alpha
$$

To see this, note that

$$
\left|g_{\theta}^{\prime}(t)\right|_{g_{\theta}(t)}^{2}=\left(\xi_{\theta}^{\prime}(t)\right)^{2}\left|g^{\prime}\left(\xi_{\theta}(t)\right)\right|_{g\left(\xi_{\theta}(t)\right)}^{2}, \forall t \in[0,1] .
$$

Hence, by (3.9),

$$
\left|g_{\theta}^{\prime}(t)\right|_{g_{\theta}(t)}^{2}=(1-2 \theta)^{-2}\left|g^{\prime}\left(\xi_{\theta}(t)\right)\right|_{g\left(\xi_{\theta}(t)\right)}^{2}, \quad \forall t \in[0,1-3 \theta],
$$

which shows

$$
\alpha_{\theta} \geq(1-2 \theta)^{-2} \frac{1}{4} \max _{s \in\left[0, \frac{1-3 \theta}{1-2 \theta}\right]} \max _{\Sigma}\left|g^{\prime}(s)\right|_{g(s)}^{2} .
$$

On the other hand, also by (3.9),

$$
\alpha_{\theta} \leq(1-2 \theta)^{-2} \alpha .
$$

Letting $\theta \rightarrow 0$, (3.11) follows from (3.12) and (3.13).

Now, by (3.10) and (3.11), we may assume

$$
\frac{1}{4} H_{o}^{2} r_{o}^{2}<\frac{\beta_{\theta}}{1+\alpha_{\theta}} \text { and } \frac{1}{4} H_{o}^{2} r_{o}^{2}<\frac{\beta_{\theta}}{1+\alpha_{\theta}}\left(1-\frac{2 m}{r_{o}}\right)
$$

for sufficiently small $\theta$. For these $\theta$, Theorem 3.1 holds for every $m_{e}$ satisfying

$$
m_{e}>m_{\theta *}
$$

where $m_{\theta *}$ is the constant in (3.5) defined via $\alpha_{\theta}$ and $\beta_{\theta}$. Now suppose $m_{e}>m_{*}$. Since $\lim _{\theta \rightarrow 0} m_{\theta *}=m_{*}$ by (3.10) and (3.11), $m_{e}$ must satisfy (3.14) for small $\theta$. Thus, the conclusion of Theorem 3.1 holds for $m_{e}$. This completes the proof of Theorem 3.1 if $g$ is not a round metric.

Finally, we consider the case that $g$ is a round metric, say $g=r_{o}^{2} g_{*}$. In this case, we have $\alpha=0, m_{*}=\mathfrak{m}_{H}(\Sigma)$, and $\left(\Sigma, g, H_{o}\right)$ is simply the boundary data of the spatial Schwarzschild metric $\gamma_{m_{*}}=d s^{2}+\bar{u}_{m_{*}}(s)^{2} g_{*}$ on $\left[s_{0}, \infty\right) \times \mathbb{S}^{2}$, where $\bar{u}_{m_{*}}\left(s_{0}\right)=r_{o}$. Now pick any $s_{1}>s_{0}$. Let $\delta>0$ be a small constant to be chosen. Similar to Lemma 2.3 in [10], we consider a smooth function $\tau$ on $\left[s_{0}, s_{1}+\delta\right]$ defined by $\tau(s)=s$ for $s \in\left[s_{0}, s_{1}\right]$, and indirectly via

$$
\tau^{\prime}(s)=1-e^{-1 /\left(s-s_{1}\right)^{2}}, s_{1} \leq s \leq s_{1}+\delta .
$$


Define a metric $\gamma_{B}:=d s^{2}+\bar{u}_{m_{*}}(\tau(s))^{2} g_{*}$. Then $\gamma_{B}$ coincides with $\gamma_{m_{*}}$ on $\left[s_{0}, s_{1}\right] \times \mathbb{S}^{2}$ and has positive scalar curvature on $\left(s_{1}, s_{1}+\delta\right] \times \mathbb{S}^{2}$ for sufficiently small $\delta$. Let $\mathfrak{m}_{H}\left(\Sigma_{s_{1}}\right)$ and $\mathfrak{m}_{H}\left(\Sigma_{s_{1}+\delta}\right)$ denote the Hawking masses of $\Sigma_{s_{1}}=\left\{s_{1}\right\} \times \mathbb{S}^{2}$ and $\Sigma_{s_{1}+\delta}=\left\{s_{1}+\delta\right\} \times \mathbb{S}^{2}$ with respect to $\gamma_{B}$, respectively. Then $\mathfrak{m}_{H}\left(\Sigma_{s_{1}}\right)=m_{*}$, and $\mathfrak{m}_{H}\left(\Sigma_{s_{1}+\delta}\right)$ can be made arbitrarily close to $\mathfrak{m}_{H}\left(\Sigma_{s_{1}}\right)$, provided $\delta$ is sufficiently small. Thus, given any fixed $m_{e}>m_{*}$, we can choose $\delta$ sufficiently small to ensure

$$
m_{e}>\mathfrak{m}_{H}\left(\Sigma_{s_{1}+\delta}\right)>0 .
$$

Moreover, we can assume $\left.\frac{d}{d s} \bar{u}_{m_{*}}(\tau(s))\right|_{s=s_{1}+\delta}>0$, i.e., $\Sigma_{s_{1}+\delta}$ has positive mean curvature with respect to $\gamma_{B}$. The theorem now again follows from Proposition 2.1.

Remark 3.1. Taking $m=0$ in Theorem 3.1, one has $k=\frac{1}{2} H_{o} r_{o}$ and

$$
\frac{1}{2} A_{o} k\left(1-k^{2}\right)=\left[\frac{\alpha\left(\frac{1}{4} H_{o}^{2} r_{o}^{2}\right)}{\beta-(1+\alpha)\left(\frac{1}{4} H_{o}^{2} r_{o}^{2}\right)}\right]^{\frac{1}{2}} \mathfrak{m}_{H}\left(\Sigma_{0}\right) .
$$

Hence, Theorem 3.1 implies Theorem 1.1 .

Remark 3.2. In Theorem 3.1, if $g$ is close to a fixed round metric $g_{*}$ in $C^{2, \eta}$-norm for some $\eta \in(0,1)$, then, by the proof of [12, Proposition 4.1], one can find a particular path $\{g(t)\}_{0 \leq t \leq 1}$ such that the associated constants $\alpha$ and $\beta$ satisfy

$$
\alpha \leq C\left\|g-g_{*}\right\|_{C^{0, \eta}(\Sigma)}^{2} \text { and } \beta \geq 1-C\left\|g-g_{*}\right\|_{C^{2, \eta}(\Sigma)}
$$

for some constant $C>0$ independent of $g$. As a result, $\alpha \rightarrow 0$ and $\beta \rightarrow 1$, as $g$ tends to $g_{*}$ in $C^{2, \eta}(\Sigma)$.

Remark 3.3. Varying $m$ subject to (3.3), Theorem 3.1 gives different estimates $m_{*}$ for the Bartnik mass $\mathfrak{m}_{B}(\Sigma)$. We refer readers to Appendix A of [12] for this optimality analysis given in a different context.

\section{REFERENCES}

[1] Arnowitt, R.; Deser, S., and Misner, C. W., Coordinate invariance and energy expressions in general relativity, Phys. Rev., 122 (1961), no. 3, 997-1006.

[2] Bartnik, R., New definition of quasilocal mass, Phys. Rev. Lett., 62 (1989), 2346-2348.

[3] Bartnik, R., Energy in General Relativity, Tsing Hua Lectures on Geometry and Analysis, International Press of Boston, Cambridge MA, (1997), 5-27.

[4] Bray, H. L., Proof of the Riemannian Penrose inequality using the positive mass theorem, J. Differential Geom., 59 (2001), no. 2, 177-267.

[5] H. L. Bray and P. T. Chrusćiel, The Penrose inequality, in: The Einstein Equations and the Large Scale Behavior of Gravitational Fields, Eds P. T. Chrusćiel and H. Friedrich, Birkhäuser Verlag, Basel, (2004), p. 39-70.

[6] Cabrera Pacheco, A. J.; Miao, P., Higher dimensional black hole initial data with prescribed boundary metric, arXiv:1505.01800.

[7] Hawking, S. W., Black holes in general relativity, Comm. Math. Phys., 25 (1972), no. 2, 152166.

[8] Huisken, G.; Ilmanen, T., The inverse mean curvature flow and the Riemannian Penrose inequality, J. Differential Geom., 59 (2001), no. 3, 353-437. 
[9] Lin, C-Y.; Sormani, C., Bartnik's mass and Hamilton's modified Ricci flow, Ann. Henri Poincaré, 17 (2016), no. 10, 2783-2800.

[10] Mantoulidis, C.; Schoen, R., On the Bartnik mass of apparent horizons, Class. Quantum Grav., 32 (2015), no. 20, 205002, 16pp.

[11] Miao, P., Positive mass theorem on manifolds admitting corners along a hypersurface, Adv. Theor. Math. Phys., 6 (2002), no. 6, 1163-1182.

[12] Miao, P.; Xie, N.-Q., On compact 3-manifolds with nonnegative scalar curvature with a CMC boundary component, arXiv:1610.07513.

[13] Shi, Y.-G.; Tam, L.-F., Positive mass theorem and the boundary behaviors of compact manifolds with nonnegative scalar curvature, J. Differential Geom. 62 (2002), no.1, 79-125.

Department of Mathematics, University of Connecticut, Storrs, CT 06269, USA. E-mail address: a.cabrera@uconn.edu

Department of Mathematics, Universität Tübingen, 72076 Tübingen, Germany.

E-mail address: cederbaum@math.uni-tuebingen.de

Institutionen för Matematik, Kungliga Tekniska hÖgskolan, 10044 Stockholm, Sweden; and School of Science and Technology, University of New England, ArmiDale, NSW 2351, Australia.

E-mail address: stephen.mccormick@une.edu.au

Department of Mathematics, University of Miami, Coral Gables, Fl 33146, USA.

E-mail address: pengzim@math.miami.edu 